\title{
Analysis of the influence of track quality for new and existing urban railway lines on train operation
}

\author{
Y. He \& Z. Li \\ College of Urban Rail Transportation, \\ Shanghai University of Engineering Science, China
}

\begin{abstract}
The difference in track quality for new and existing urban railways influences comfort and security in train operation. In this paper, the track quality of two typical urban railway lines in one city was studied. The Sperling index, which was obtained based on data collected from a self-made accelerometer fixed on the operating vehicle, together with track geometry data, were used to determine the influence of existing and new lines track condition on train operation. The results show that peak and Sperling values for the new urban railway is lower and achieves the qualified standard, while existing urban railway exceeds the standard. The quality state of horizontal operation for both lines is worse than that of vertical. The Track Quality Index (TQI) can reflect the quality state of train operation to a certain degree, and then maintenance is recommended to be carried out for the different urban railway conditions.
\end{abstract}

Keywords: urban rail transportation, track, Sperling, TQI, maintenance.

\section{Introduction}

In recent years, the subway has rapidly developed as a major infrastructure, and it has improved people's travel conditions and quality of life. Because the planning and construction of the subway is a huge systematic engineering project, and the formation of a line network is very time consuming work, the track condition of the new subway lines is inevitably different from the existing ones. To improve the comfort and security of train operation effectively, it is necessary to study the track quality of the new and existing subway lines to improve the 
level of the entire subway line management. Currently, a lot of research has focused on the running state of vehicles but none of them concerned the track structure. The relationship between the actual vibration inside a metro vehicle, the range of the vibrating frequency and the speed of the metro vehicle was studied in [1], and the ride index of a metro vehicle was analyzed in [2]. These researches mostly focused on special line and track structure, but less on the influence of track state on subway vehicle running status. Vehicle running comfort of an overhead railway and straddle-type monorail was analyzed in $[3,4]$. The influence of a floating slab track on metro vehicle vibration was analyzed in [5]. In summary, these researches are very important to vehicle and track structure design, but there is little research about the comparison between new and existing subway lines. In this paper, the track quality of two typical subway lines in one city was studied, and the Sperling index was analyzed based on the data collected from a self-made accelerometer fixed to the operating vehicle. At the same time the track geometry was added to analyse the influence of the existing and new lines track condition on train running state. This research can be used to guide subway maintenance and repair in future.

\section{Vehicle ride vibration test}

\subsection{Test lines and time}

The existing subway line is $50 \mathrm{~km}$ long with all electric underground ballastless track, with 26 stations and has been in operation since 2000 .

The new subway line is $27 \mathrm{~km}$ long with all electric underground ballastless track, with 16 stations and has been in operation since 2010 .

To compare track condition data, a vehicle ride vibration test was conducted within 2 or 3 days after the track inspection car on line detection. Because the track state will not change in a short time, the acceleration of the vehicle body vibration and track state were assumed to have a higher matching degree.

\subsection{Test sites and instruments}

Vehicle vibration acceleration measuring points were determined by related standards, measuring point 2 was fixed on the floor above the vehicle bogie center plate [6]. For comparison, measuring point 1 was fixed by the door near the carriage and measuring point 3 was fixed on the train connections, as shown in Figure 1. In all test lines, a type "A" vehicle was used.

Type 891 sensors and type 891 six line amplifiers were used as the test equipment. The signal was collected by a self-made real time monitoring system (shown in Figure 2), with $500 \mathrm{~Hz}$ sampling frequency. Due to the crowded and overloaded operation of the subway network in peak hours, which causes difficulties in instrument layout, this test was conducted during the off-peak time. 


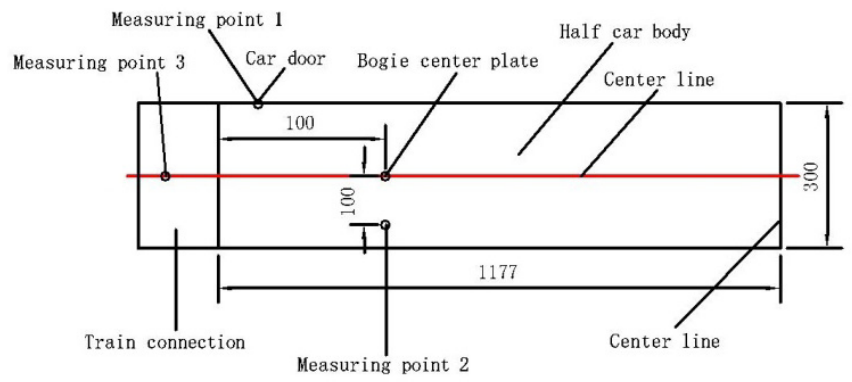

Figure 1: Layout of measuring points (unit: $\mathrm{cm}$ ).

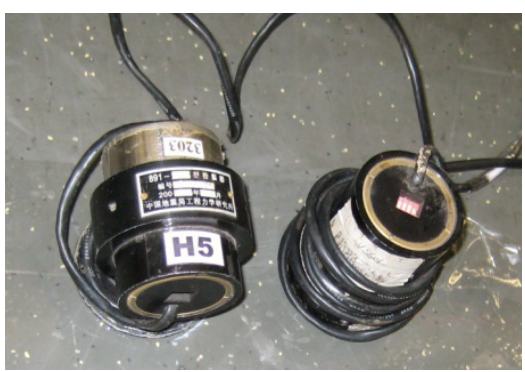

(a) Type 891 sensors.

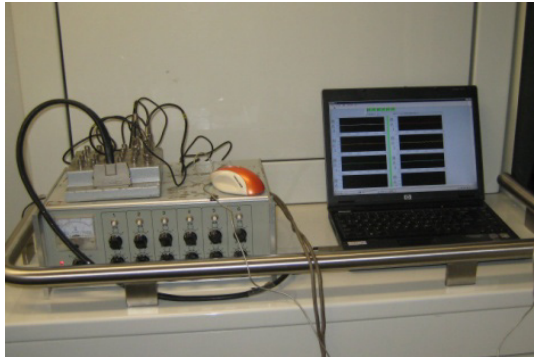

(b) Type 891 six line amplifiers.

Figure 2: Testing instrument and monitor system.

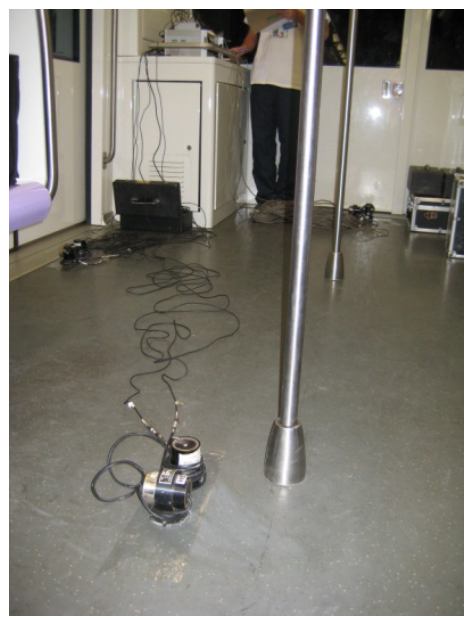

Figure 3: Layout of testing field (unit: $\mathrm{cm}$ ). 


\section{Operation comfort analysis of a subway vehicle}

\subsection{Analysis of the acceleration time domain peak}

The horizontal and vertical vehicle vibration acceleration is used to evaluate the vehicle operation comfort state by the department of the railway management in China, and this is further classified into a four-grade standard to determine the vehicle operating comfort state [7]. In this method, the acceleration index is taken as half of the actual amplitude peak value. The acceleration indexes of new and existing subways lines for one typical interval are shown in Figure 4 and Figure 5.

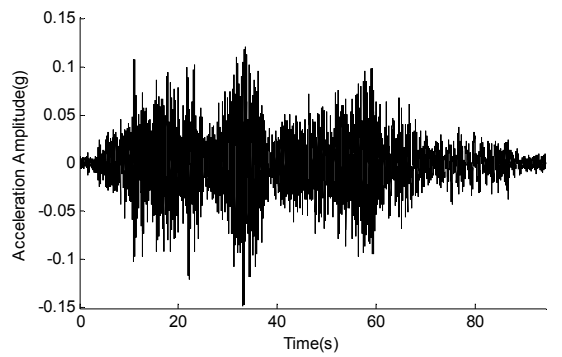

(a) Horizontal acceleration waveform.

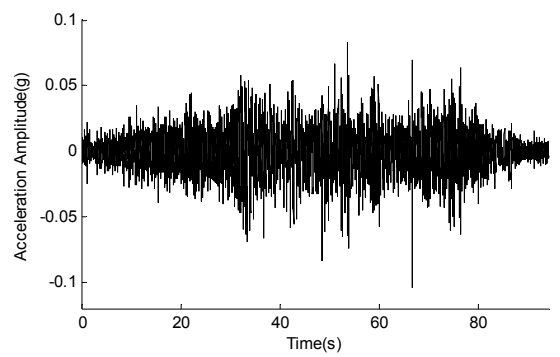

(b) Vertical acceleration waveform.

Figure 4: Vehicle vibration acceleration waveform of new line typical intervals.

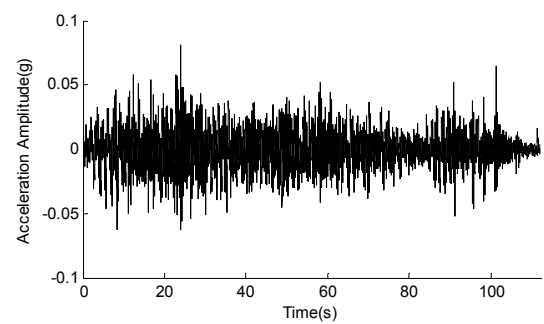

(a) Horizontal acceleration waveform.

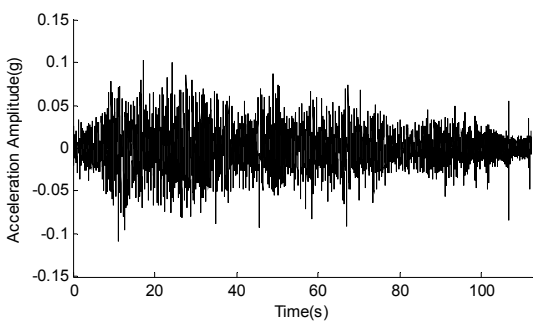

(b) Vertical acceleration waveform.

Figure 5: Vehicle vibration acceleration waveform of existing line typical intervals.

As shown in Figures 4 and 5, it was found that the metro vehicle experienced the phases of start-up, acceleration, deceleration, normal speed and station stopping in the interval, Hence, it was necessary to pick up the acceleration index of the phase of normal speed in the running interval to analyze the vehicle operation comfort state. 
The distribution of acceleration amplitude for the new and existing lines is further calculated by the acceleration amplitude statistical method. Figures 6 and 7 illustrate the vibration acceleration amplitude statistic of the existing and new urban railway. In the figure, the corresponding management limits are also given for comparison. As shown in Figure 6, for the new subway lines, the amplitude of vibration acceleration above the vehicle bogie (point 2 ) is relatively large. In some intervals the value is over the moderate management limit, and the number of vertical intervals in which acceleration amplitude is over the limit is more than the horizontal intervals. It was then suggested that more attention should be given to profile irregularity for new lines, especially for shortwave irregularity, which is the main reason to induce vehicle vertical vibration amplitude over the limit. Hence, it is necessary to take a pre-grind rail method to eliminate profile irregularity. In Figure 7, for the existing subway lines, it is shown that the amplitude of horizontal vibration acceleration on the vehicle conjunction (point 3 ) is relatively large, and the amplitude of vertical vibration acceleration recorded in the three measured points has no significant regularity. However, the amplitude of horizontal and vertical acceleration in many intervals is over the limit. The situation of over the limit in vertical intervals is worse than that in horizontal intervals. Comparing the new and existing subway lines, the

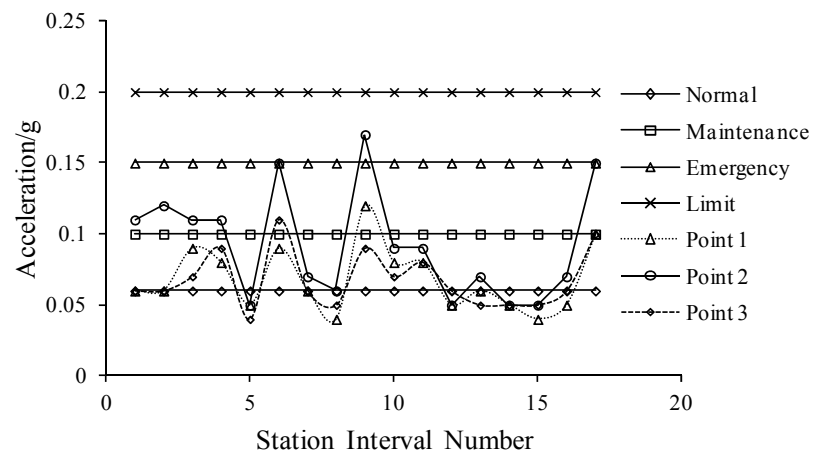

(a) Horizontal acceleration.

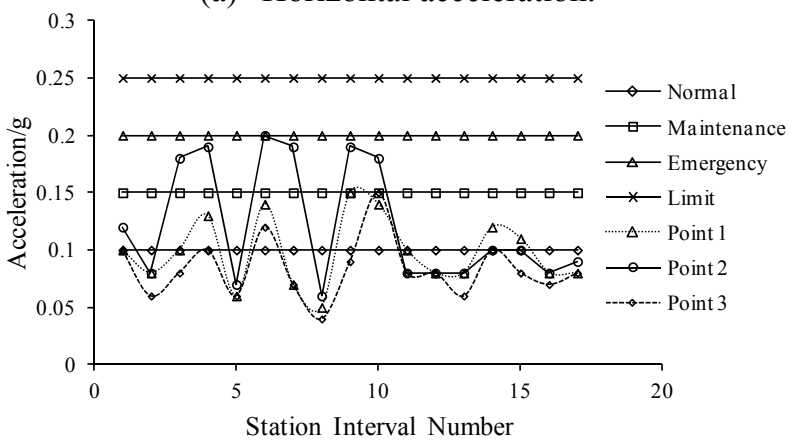

(b) Vertical acceleration.

Figure 6: Vibration acceleration amplitude statistic of new urban railway (unit: g). 
amplitude distribution characteristics for both lines are largely different. This is mostly because the existing lines were built much earlier, and contain many small radius curves, which may cause serious wear. At the same time, track overall settlement may cause many line problems, which seriously influence the alignment ride condition. However, it should be noted that there is no standard vehicle vibration acceleration evaluation index for urban railway lines, hence it may be debatable if the national railway evaluation index is suitable.

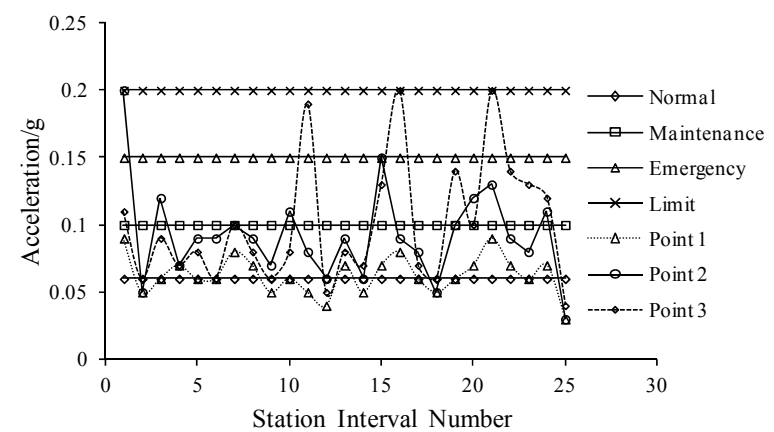

(a) Horizontal acceleration.

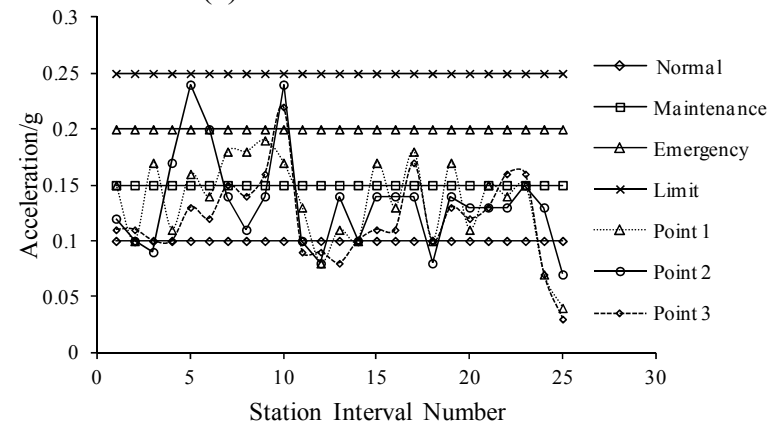

(b) Vertical acceleration.

Figure 7: Vibration acceleration amplitude statistic of existing urban railway (unit: g).

\subsection{Sperling index}

The main factor for evaluating passengers' feeling about the vibration environment is when the vehicle is stationary. Sperling index W is employed to calculate the index of vehicle stationary. The index $\mathrm{W}$ is defined as follows:

$$
W=\left(W_{1}^{10}+W_{2}^{10}+\cdots+W_{n}^{10}\right)^{1 / 10}
$$

where $W_{i}$ is the stationary value corresponding to the frequency defined by:

$$
W_{i}=7.08\left[\frac{A_{i}^{3}}{f_{i}} F\left(f_{i}\right)\right]^{1 / 10}
$$


where $A_{i}(\mathrm{~g})$ is the acceleration amplitude corresponding to the frequency $f_{i}$ from the vibration signal Fourier transformed, and $F\left(f_{i}\right)$ is the correction coefficient for $f_{i}$.

There are three ratings of Sperling Index for railways, as shown in Table 1.

Table 1: Sperling level assessment.

\begin{tabular}{|c|c|c|}
\hline Sperling level & Condition & $\begin{array}{c}\text { Assessment } \\
\text { standard }\end{array}$ \\
\hline Level 1 & Excellent & $<2.50$ \\
\hline Level 2 & Good & $2.50-2.75$ \\
\hline Level 3 & Qualified & $2.75-3.00$ \\
\hline
\end{tabular}

The Sperling index $\mathrm{W}$ for the above new and existing urban railway lines in this study is shown in Figures 8 and 9.

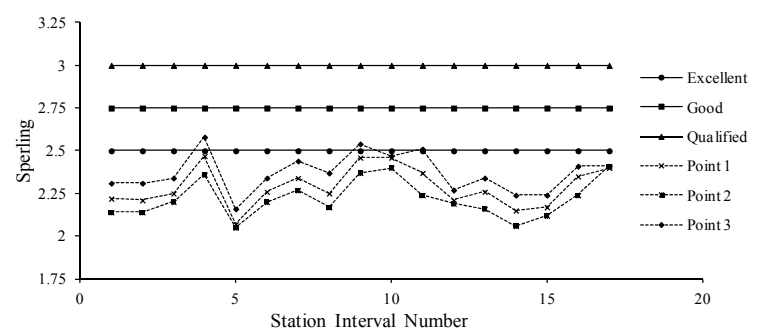

(a) Horizontal Sperling.

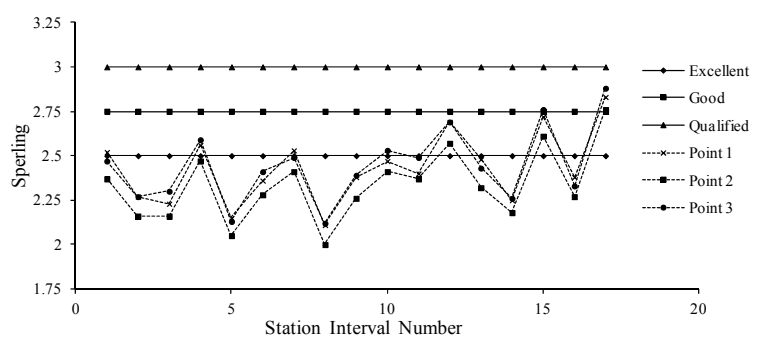

(b) Vertical Sperling.

Figure 8: Sperling of new urban railway.

Figure 8 , for the new urban railway, shows that the Sperling values in most of the intervals are qualified and even excellent, and there is no difference in horizontal and vertical when the vehicle is stationary. The Sperling value for measuring point 2 fixed on the floor above the vehicle bogie center plate is the lowest. As shown in Figure 9, for the existing urban railway, the Sperling values 
in 20 percent of vertical intervals and 30 percent of horizontal are within the standard of excellent, and Sperling value for measuring point 2 in some intervals reached 3. Compared with these results, it was found that the stationary vehicle for the existing urban railway is in a worse condition than that for the new urban railway line, and the results from peak analysis show the same trend. Further, the state of the horizontal interval in the stationary vehicle is worse than that of the vertical. Hence, it is concluded that alignment irregularity, curve lines and super-elevation of the railway should be paid more attention in maintenance, and the frequency of maintenance of the existing urban railway lines should be higher than that of new lines.

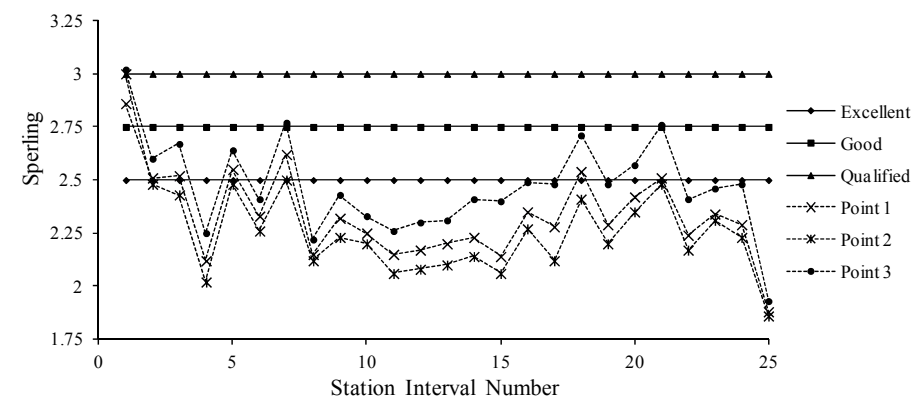

(a) Horizontal Sperling.

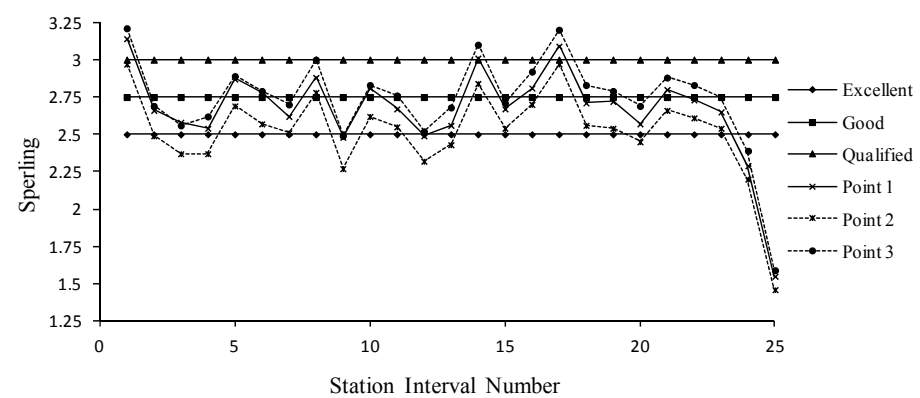

(b) Vertical Sperling.

Figure 9: Sperling of existing urban railway.

\section{Analysis of TOI and Sperling index}

TQI was proposed to evaluate the general quality condition of the track in the early 90 s by the China Academy of Railway Sciences. This method takes $200 \mathrm{~m}$ as a unit interval, in which standard deviation $\sigma$ was calculated for the eight single index of track irregularity including left profile, right profile, left alignment, right alignment, gauge, cross level and warp irregularity. TQI is then defined by summing up each single index, and reflects the discrete degree of the track geometry irregularity amplitude in every unit. 
In order to analyze the influence of the TQI on the operation of the vehicle when stationary, regression analysis was conducted on the Sperling Index and TQI of every track geometry irregularity according to the track irregularity measuring data and operating trains vibration acceleration test data from the new and existing urban railway for each $200 \mathrm{~m}$ interval. The results are shown in Figures 10 and 11.

It is shown in the figures that the horizontal Sperling and alignment TQI for both the new and existing urban railway have a strong relativity of 0.18 and 0.061, respectively. Vertical comfort and profile TQI for the new urban railway show a strong relativity of 0.054 , but vertical comfort and every single and general TQI for the existing urban railway shows negative relativity. Due to the limitation of test conditions and the complexity of the factors influencing vehicle vibration acceleration, the TQI and Sperling index show weak relativity. It was also found that some single TQI and Sperling index have opposite relativity, which means TQI reflects train operation comfort state to a certain degree. Hence, a more reasonable and accurate index is needed to obtain a better evaluation of the train operation comfort state.

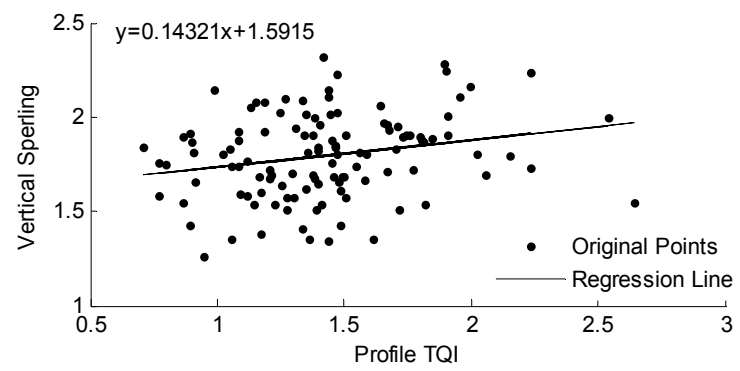

(a) Profile TQI and vertical Sperling.

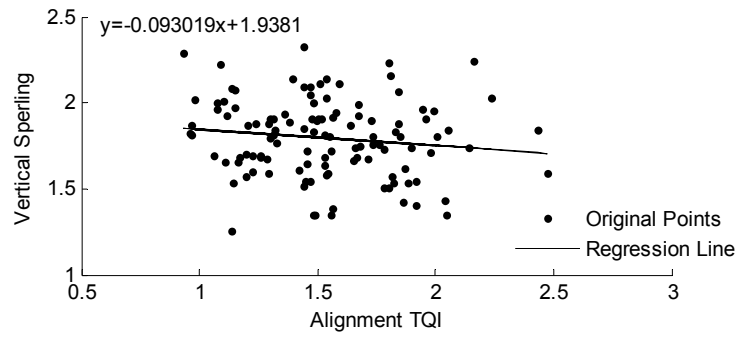

(b) Alignment TQI and horizontal Sperling.

Figure 10: Regression analysis with TQI and Sperling of the new urban railway. 


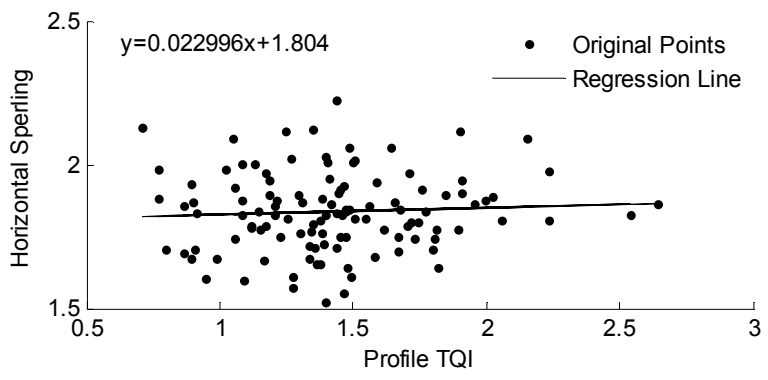

(a) Profile TQI and vertical Sperling.

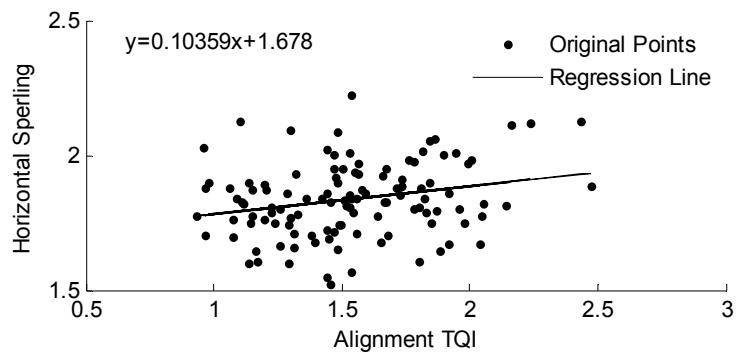

(b) Alignment TQI and horizontal Sperling.

Figure 11: Regression analysis with TQI and Sperling of the existing urban railway.

\section{Conclusions and recommendations}

The influence of the new and existing urban railway quality state on train operation comfort was analyzed by self-made acceleration sensors installed in the train. The Sperling test and regression analysis with TQI were conducted. The results show that:

(1) For the new urban railway lines, the amplitude of vibration acceleration above the vehicle bogie is relatively large. In some intervals these values are over the moderate management limit and the number of vertical intervals in which acceleration amplitude is over the limit is more than that in horizontal intervals. For the existing subway lines, the amplitude of horizontal vibration acceleration on vehicle conjunction is relatively large, and in many intervals these values are over the limit. The situation of over the limit in vertical intervals is worse than that in horizontal intervals. Compared with the new and existing urban railway lines, amplitude distribution characteristics for these two lines are very different. Maintenance should be performed and planned for the different urban railway conditions.

(2) The Sperling value for the new urban railway shows that most of the intervals are qualified and even excellent, and there is no difference between horizontal and vertical. For the existing urban railway, the Sperling values in 
20 percent of vertical intervals and 30 percent of horizontal are within the excellent standard, and the Sperling value of measuring point 2 in some intervals is over 3. Overall, the stationary vehicle of the existing urban railway is in a worse condition than that of the new urban railway line.

(3) Due to the limitations of the test conditions and the complexity of the factors influencing vehicle vibration acceleration, the TQI and Sperling index show weak relativity. Some single indexes of TQI and the Sperling index show opposite relativity, which means that TQI reflects train operation comfort state to a certain degree. Hence, a more reasonable and accurate index is needed for better evaluation of train operation comfort state.

\section{Conflict of interests}

All of the authors do not have any direct financial relation with the commercial identities mentioned in this paper.

\section{Acknowledgements}

This work was supported by the Key Subject Building Project of Shanghai Municipal Education Commission (J51401), the Innovation Program of Shanghai Municipal Education Commission (14YZ137), and the Training Project of Youth College Teachers of Shanghai Municipal Education Commission (ZZGJD13041).

\section{References}

[1] Huang Shouyu, Cao Guangzhong, Zhang Hua,et al. Measurement of Vibration inside Metro Vehicles [J]. Urban Mass Transit, 2011, 14(4): 99-102.

[2] Liu Liying, Zhou Wenxiang, Xu Na. Accuracy Analysis of Ride Index for Railway Vehicle [J]. Electric Locomotives \& Mass Transit Vehicles, 2008, 31(1): 38-40.

[3] Zhu Jinlong. Test and Analysis of the Cozy Extent of the overhead Railway Vehicles [J]. Urban Mass Transit, 2003, 6(2): 46-51.

[4] Ma Jibing, Pu Qianhui, Xia Zhaoguang. Test and Evaluation of the Comfort of Passengers in Straddle-type Monorail Vehicles [J]. Urban Rapid Rail Transit, 2006, 19(6): 46-50.

[5] Li Zaiwei, Lian Songliang, Liu Xiaozhou. Measurement and analysis of influence of floating slab track on metro vehicle vibration [J]. Urban Mass Transit, 2013, 16(9): 23-26.

[6] GB/T 5599-1985. Railway vehicles; Specification for evaluation the dynamic performance and accreditation test [S]. 1985.

[7] Xu Yude, Li Haifeng, Dai Yuehui. Track maintenance \& management for urban mass transit [M]. Shanghai: Tongji University Press, 2007. 
[8] Li Zaiwei, Lian Songliang, Liu Xiaozhou. Time-frequency Analysis of Vehicle-Track vertically Coupling System Based on Hilbert-Huang Transform. Journal of Vibration, Measurement \& Diagnosis, 2013, 33(5): 799-803.

[9] Zaiwei Li, Songliang Lian, and Yuelei He. Time-Frequency Analysis of Horizontal Vibration for Vehicle-Track System Based on Hilbert-Huang Transform [J]. Advances in Mechanical Engineering, vol. 2013, Article ID 954102, 5 pages, 2013. doi: 10.1155/2013/954102. 